\title{
Cellular diversity in a snapshot
}

A new platform detains single cells in picoliter wells to generate transcriptional profiles in large numbers.

Sequencing has historically been applied to bulk samples, which causes information from individual cells to be averaged - but it wasn't this way by design. "I think that's just a fluke of how the technology has developed over the last 20 years," says Stephen Fodor, founder of Cellular Research, Inc. The technical challenges of dealing with little starting material are now being overcome, yet another challenge has been how to sample enough cells to represent the population. As a founder of DNA microarray manufacturer Affymetrix, Fodor has experience in scaling up assays. With first author Christina Fan and Glenn Fu at Cellular Research, he has developed a way to profile gene expression in thousands of single cells at once.

Their new CytoSeq platform consists of 20-picoliter wells that are sparsely loaded so that most wells randomly receive either one cell or none. Beads sized to fit one per well are then added at high density, and cells are lysed. Cellular RNA hybridizes to each bead, which is functionalized with a unique barcode to identify the cell and with millions of molecular barcodes to label each RNA molecule for accurate quantification of gene expression by digital counting. The beads are then pooled, and reverse transcription, PCR and sequencing library preparation take place in a single tube. Approximately $20 \%$ of transcripts in each cell are detected by sequencing.

Targeting around 100 genes for sequencing, the researchers used CytoSeq to classify human blood cells into discrete cell types and found rare antigen-specific T cells from virus-infected individuals. Although the team is developing a whole-transcriptome assay, they note that assaying many cells requires shallow sequencing to be practical, which can bias measurements in favor of the most abundant genes.

Scale is important. When studying T-cell activation, they found that fewer than $0.1 \%$ of cells contributed to two-orders-ofmagnitude increases in interleukin expression at the population level. "Without doing single-cell analysis at that high scale, you won't get to see that information," says Fan. CytoSeq can scale up to hundreds of thousands of cells.

Cellular Research is planning a full product launch for next year. The hope is that an easy-to-use and scalable platform will help researchers switch to studying gene expression at single-cell resolution. "This is the sort of mind change that is coming," says Fodor.

\section{Tal Nawy}

\section{RESEARCH ARTICLES}

Fan, H.C. et al. Combinatorial labeling of single cells for gene expression cytometry. Science 347 , 1258367 (2015). 